\title{
First Dorsal Metacarpal Artery Flap Versus Reverse Homodigital Dorsoradial Flap in Soft Tissue Reconstruction of Post-Traumatic Deep Thumb Defects
}

\author{
AYMAN FIKRY MEHANNA, M.D.; MOHAMED ALI NASR, M.D. and MOHAMED WAHSH, M.D. \\ The Department of General Surgery, Plastic \& Reconstructive Surgery Unit, Faculty of Medicine, Zagazig University
}

\begin{abstract}
Background: Soft tissue reconstruction of thumb defects is of paramount importance to keep the safety of deeper structures and preserve the function of thumb. Proper selection of flap coverage must provide a versatile, pliable coverage with restored thumb motor function and sensibility in pulp area, in one stage procedure with acceptable aesthetic outcome in both donor and defect areas. The aim of this study was to compare the use of two regional flaps $1^{\text {st }}$ dorsal metacarpal artery flap versus the reverse homodigital dorsoradial artery flap in reconstruction of partial soft tissue defects in the thumb as regard the function, sensibility and aesthetic outcome.
\end{abstract}

Patients and Methods: Eighteen patients had been included in this prospective study was divided randomly in two groups $\mathrm{A}$ and $\mathrm{B}$, they undergone the procedures. In the two groups; the flap dimensions, and operative time were recorded intraoperatively. Postoperatively; the motor function, two point discrimination testing for sensibility, the complications, and the aesthetic outcome were assessed during the follow-up period.

Results: All flaps in both groups were survived, venous congestion was the main complications, which was more occurring in group B (3 cases) than group A (one case). There was statistically significant difference regarding the flap dimensions and operative time between the two groups. Restored motor function and sensibility were satisfactory in both groups. Aesthetically; the redial scars were more appealing in group B than A.

Conclusion: Beside the well-known $1^{\text {st }}$ DMCA flap, the RHDDRA flap could be a useful alternative in thumb reconstruction with less operative time, larger skin island dimensions and more attractive residual scars.

Key Words: Thumb reconstruction - First dorsal metacarpal artery - Dorsal radial digital artery.

\section{INTRODUCTION}

In complex thumb injuries with large skin and soft tissue substance loss, exposure of deep vital structures such as bone, tendon, and nerve could happen [1]. These skin defects could not be left for healing by secondary intention and are not amena- ble to simple closure or skin graft coverage [2], in this situation the need of immediate coverage with a flap is mandatory to preserve the deep vital structures [3].

Several flaps have been proposed for thumb defect resurfacing ranging from local, regional to free flaps [3,4]. the choice of a particular flap must fulfill the unique reconstruction goals concerning with soft tissue coverage of thumb defect, which should be stable, pliable to the extent not to hinder mobility of both donor and recipient site, maintain length without bone shortening [5.6], beside obtaining a proper soft tissue padding with restoration of the contour and protective sensibility in reconstructed pulp injuries [2,4].

First dorsal metacarpal artery flap (1 ${ }^{\text {st }}$ DMCA) has stood the test of time and is considered as the workhorse for partial thumb defect reconstruction, but in certain situations it could be not selected such as; this flap is no longer available due to associated injury to its vascular pedicle, or it is blamed as it is a heterodigital flap with tethering and morbidity to adjacent index finger [4].

An alternative flap may be needed, many anatomical studies have defined the dorsal blood supply of the thumb, revealed a constant anatomy of dorsoradial digital artery of the thumb; this led to the development of a reverse homodigital dorsoradial fasciocutaneous flap from the dorsum of the thumb metacarpal area [7].

The purpose of this study was to compare the use of two regional flaps; first dorsal metacarpal artery [1 ${ }^{\text {st }}$ DMCA], and reverse homodigital dorsoradial artery flap [RHDRA] for reconstruction of partial soft tissue defects of the thumb, by assessing wound healing of donor and reconstructed sites, sensibility recovery in reconstructed pulp 
area, and functional outcome of the reconstructed thumb.

\section{PATIENTS AND METHODS}

From September 2016 to October 2017, Random sample of eighteen patients having partial thumb soft tissue defect were included in this study. The patients were equally divided into two groups; nine patients were representing the odd numbers, were operated by 1 st dorsal metacarpal artery flap and described as group A and nine patients were represented the even numbers, were operated by reverse homodigital dorsoradial artery flap and described as group B.

This study was conducted in plastic surgery unit, general surgery department, Zagazig University after approval of The Ethical Committee in
Zagazig University; all participating patients were informed about the steps of the procedure and possible complications and were consented. $\mathrm{Pa}-$ tient's details are summarized in Table (1).

The patients with a mutilating hand trauma, in which there is an associated injury that may compromise the vascular pedicle or the cutaneous territory of both flaps, were excluded from the study.

In both groups; all patients received perioperative antibiotic, the surgical procedures were done under general anesthesia. After wound debridement of the thumb defect or (post burn-post traumatic) contracture scar release, flap harvesting and mobilization of the pedicle was performed under tourniquet control and loupe magnification.

Table (1): Patients data.

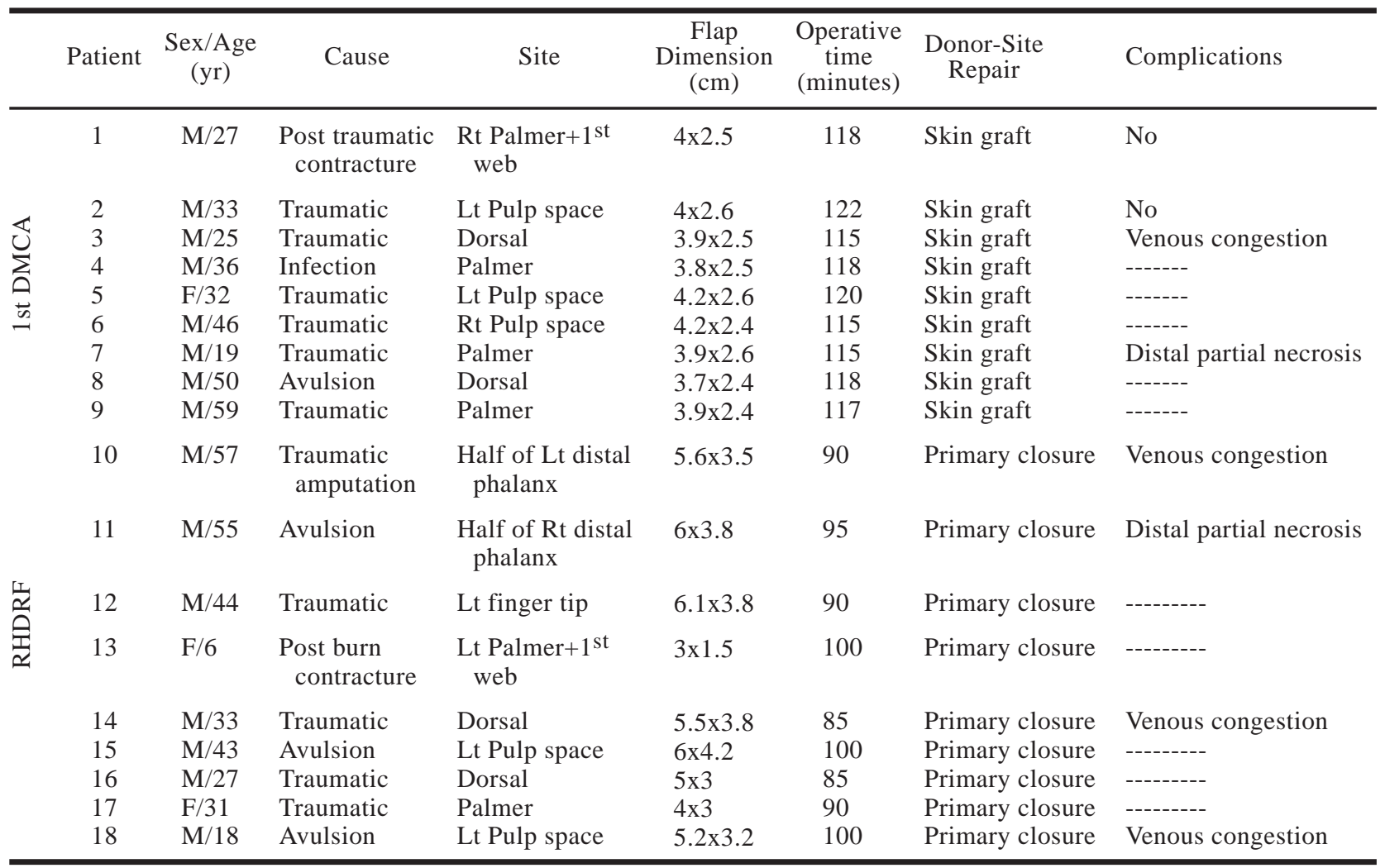

First dorsal metacarpal artery (DMCA) flap:

\section{Surgical anatomy:}

It is a pedicled neurovascular flap based on 1 st dorsal metacarpal artery with its concomitant veins and a branch of the superficial radial nerve [8]. This artery constantly originates from the radial artery just distal to the extensor pollicis longus tendon and proximal to the point at which the radial artery pierces between the two heads of the first dorsal interosseous muscle, and then it runs distally either in fascial, subfascial, or mixed course in relation to the fascial layer of the first dorsal interosseous muscle. 
It divides at the middle of the second metacarpal bone into three terminal branches; The radial branch goes to the thumb, while the intermediate branch runs to the first web space, and the ulnar branch to the index finger which runs distally within a musculo-osseous groove, between the ulnar head of the $1^{\text {st }}$ dorsal interosseous muscle and the radial shaft of the $2^{\text {nd }}$ metacarpal bone, it usually terminates at the level of the metacarpo-phalangeal joint and then arborizes into the dorsal skin of the index finger after giving off a perforating branch at the level of the metacarpal neck to join the second palmar metacarpal artery $[\mathbf{9 , 1 0 ]}$.

\section{Surgical procedure:}

With the aid of Doppler $8 \mathrm{MHz}$ the origin and the course of the artery is marked, then the flap cutaneous territory is outlined between the MP joint and the proximal interphalangeal joint. Start raising of the skin paddle from the distal to the proximal direction, cautiously leaving the paratenon of extensor tendon undisturbed to guarantee skin graft take and the allowing free gliding of the tendon. A lazy S skin incision is done over the fascial pedicle with skin subdermal dissection, Safe dissection of the fascial pedicle can be achieved by including the radial side of the periosteum of the shaft $2^{\text {nd }}$ metacarpal bone, and then continuing the flap raising in the subfascial plane with including a strip of the interosseous muscle fascia and the sensory branch of the radial nerve with the pedicle, the tourniquet is deflated to check flap vascularity, Figs. $(1,2,3)$.

A wide subcutaneous tunnel is made, and the flap is transferred by gentle traction into the defect area of the thumb and in sited. The donor site is grafted with full-thickness skin graft. Finally thumb is splinted for 2 weeks.

\section{Reversed homodigital dorsoradial artery $(\mathrm{RH}-$ DRA) flap:}

\section{Surgical anatomy:}

It is based on the dorsal radial digital collateral artery, it originates from the radial artery at the level of the anatomical snuffbox; it passes under the extensor pollicis brevis tendon, then it has a straight and regular course on a subcutaneous plane along the radial side of the thumb, it constantly communicates with the palmar circuit at the level of the middle third of the proximal phalanx. In its course along the diaphysis of the $1^{\text {st }}$ metacarpal bone, it accompanies the dorsal collateral branch of the radial nerve [3].

\section{Surgical procedure:}

With the aid of Doppler $8 \mathrm{MHz}$ the origin and the course of the artery is identified till the pivot point at the middle of the proximal phalanx, a line is drawn along its course. The predicted skin island is centered on that line; its dimensions nearly equal the defect size. Skin incision is done at the periphery of the skin island, and at the site of anticipated subcutaneous pedicle; a very superficial lazy-S skin incision is done extending from the distal end of skin island to the pivot point with sub-dermal dissection is done to expose the pedicle, and enough quantity of soft tissue is left around the pedicle to protect the vascular axis and allows venous outflow.

Then the flap is raised in a proximal to distal direction, during raising the flap, the extensor pollicis brevis is identified, and it is gently spread away. The sensory collateral nerve is identified, and it enclosed in the flap. The pedicle is dissected from the deep tissues; it stopped near to the pivot point to protect the anastomosis with the perforating digital palmar vessels.

The bridging skin between the pivot point and the edge of the defect is incised and elevated; the tourniquet is released to ensure proper blood flow to the flap, then the flap can be transposed and sutured at the tissue defect site. The skin above the pedicle is left partially opened without stitches to avoid any tension on the pedicle. Finally the donor site can be primarily closed directly and the thumb is immobilized for two weeks, Figs. $(4,5,6)$.

Follow-up: all patients were followed-up at a period ranging at least from 6 weeks up to 6 months. During the follow-up period the flaps were examined for sensation, thumb movement (flexion, extension and opposition), and post-operative cosmetic results in both donor and recipient sites.

\section{RESULTS}

All flaps in both groups were survived, with minimal venous congestion in only one case in group A (compression by a narrow tunnel), and three cases in group B; nothing was done more than removal of stitches over the pedicle to relive the compression, and one case in each group with partial marginal necrosis which eventually healed spontaneously. Otherwise all patients had maintained full flexion and extension of the thumb, and the outcome was satisfactory in all cases but the redial scars were more appealing in group B than A. 
There was statistically significant difference between group A and group B regarding flap dimensions $(\mathrm{cm})$ with $(p$-value 0.008$)$, the mean value was [3.9x2.5] in 1 ${ }^{\text {st }}$ DMCA flap group while it was [5.2x3.3] with RHDRA flap group. Also there was statistically significant difference between the two studied groups regarding operative time (minutes) with ( $p$-value 0.000$)$, the mean value was [117.56] in group A while it was shorter [92.78] with group B.
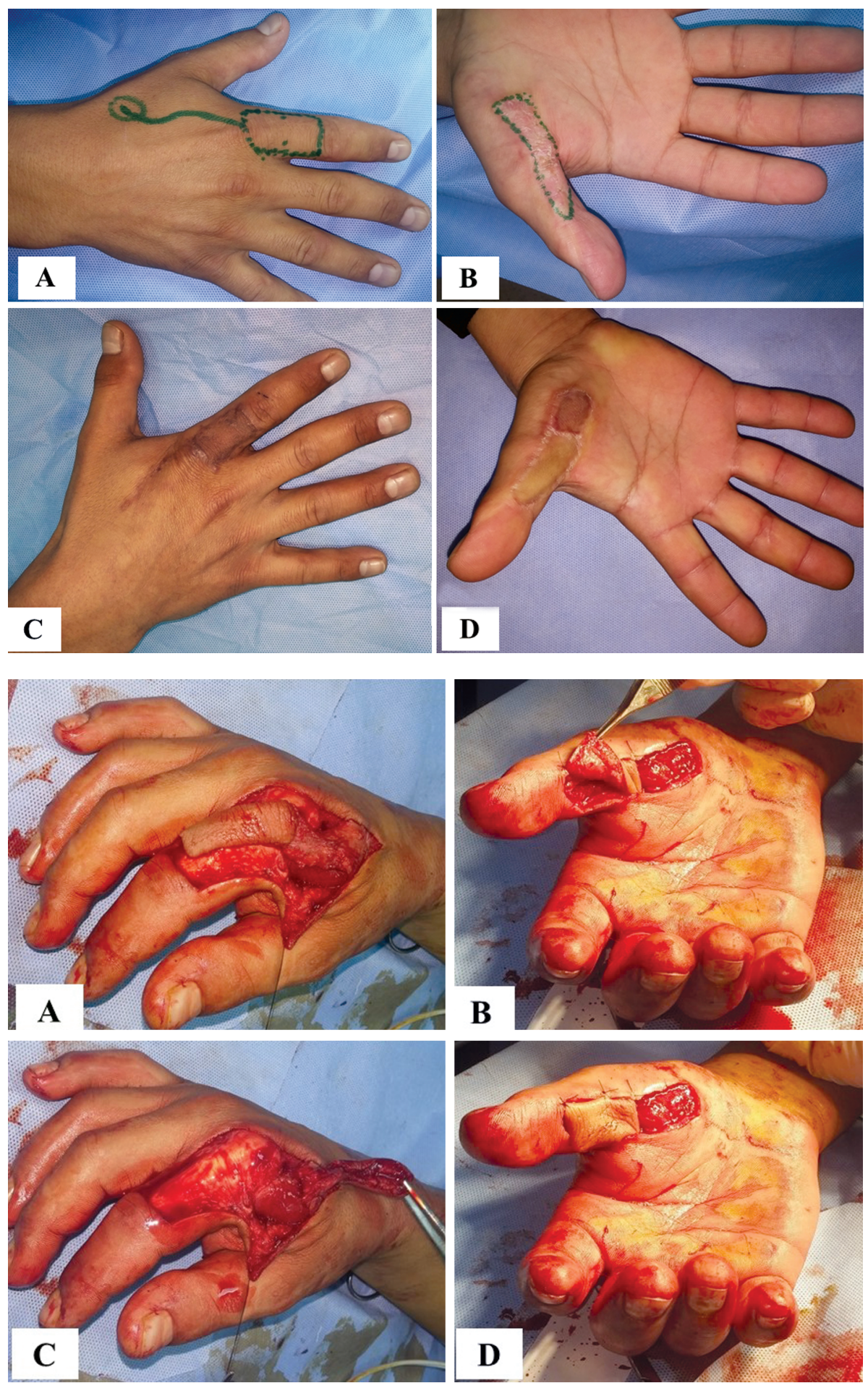

Fig. (1): (A) Preoperative marking, (B) The post traumatic contracture, (C) Post-operative view of donor scar 3 months later, (D) Postoperative view of recipient site 3 months later.

Fig. (2): (A) $1^{\text {st }}$ DMCA Skin island an pedicle harvesting, (B) Skin island at the recipient area, (C) Subfascial dissection of the pedicle, (D) Flap insitting. 

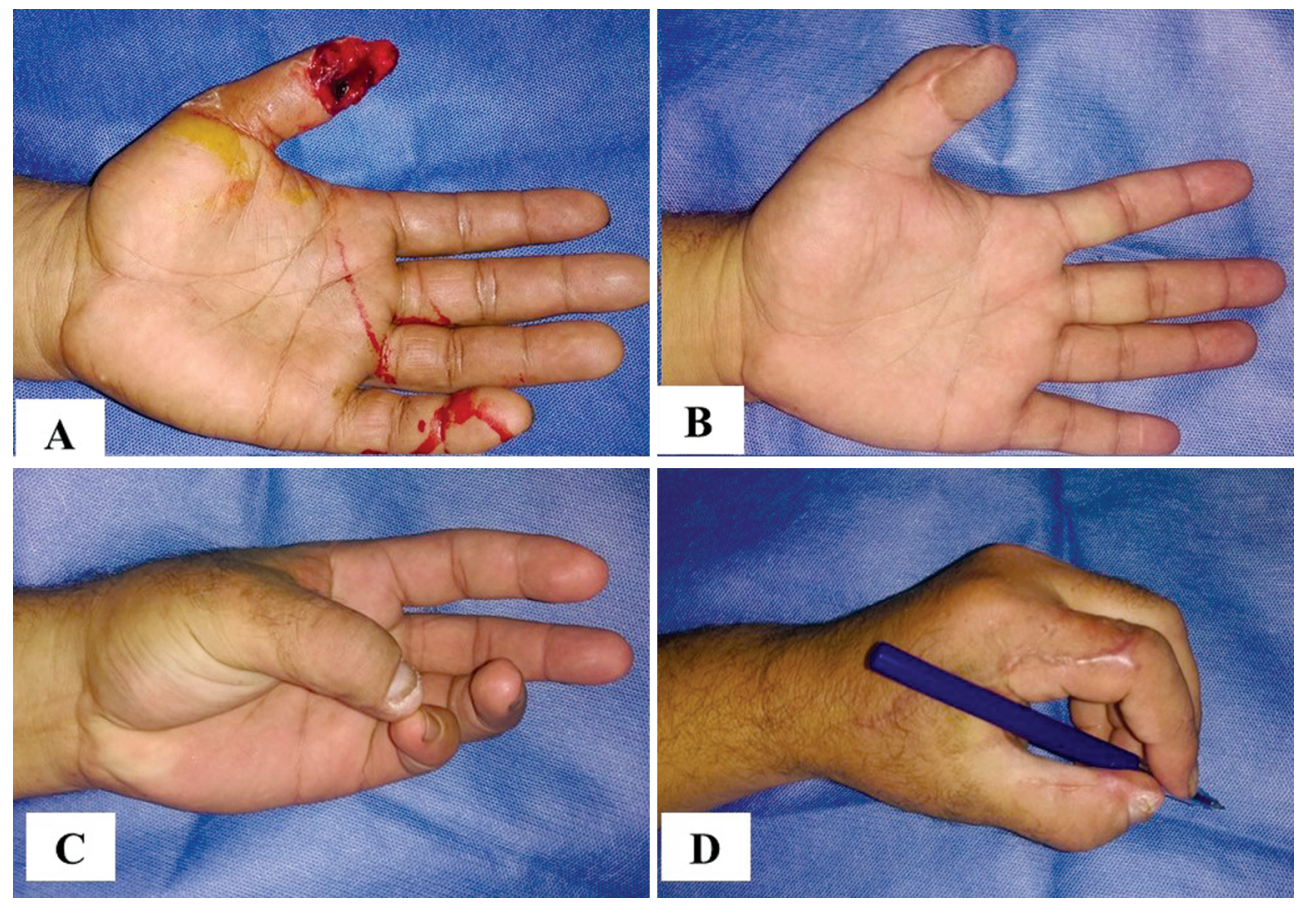

Fig. (3): (A) Preoperative fingertip injury, (B) Post-operative view of $1^{\text {st }}$ DMCA flap, (C) Opposition of the thumb, (D) Full function of thumb.
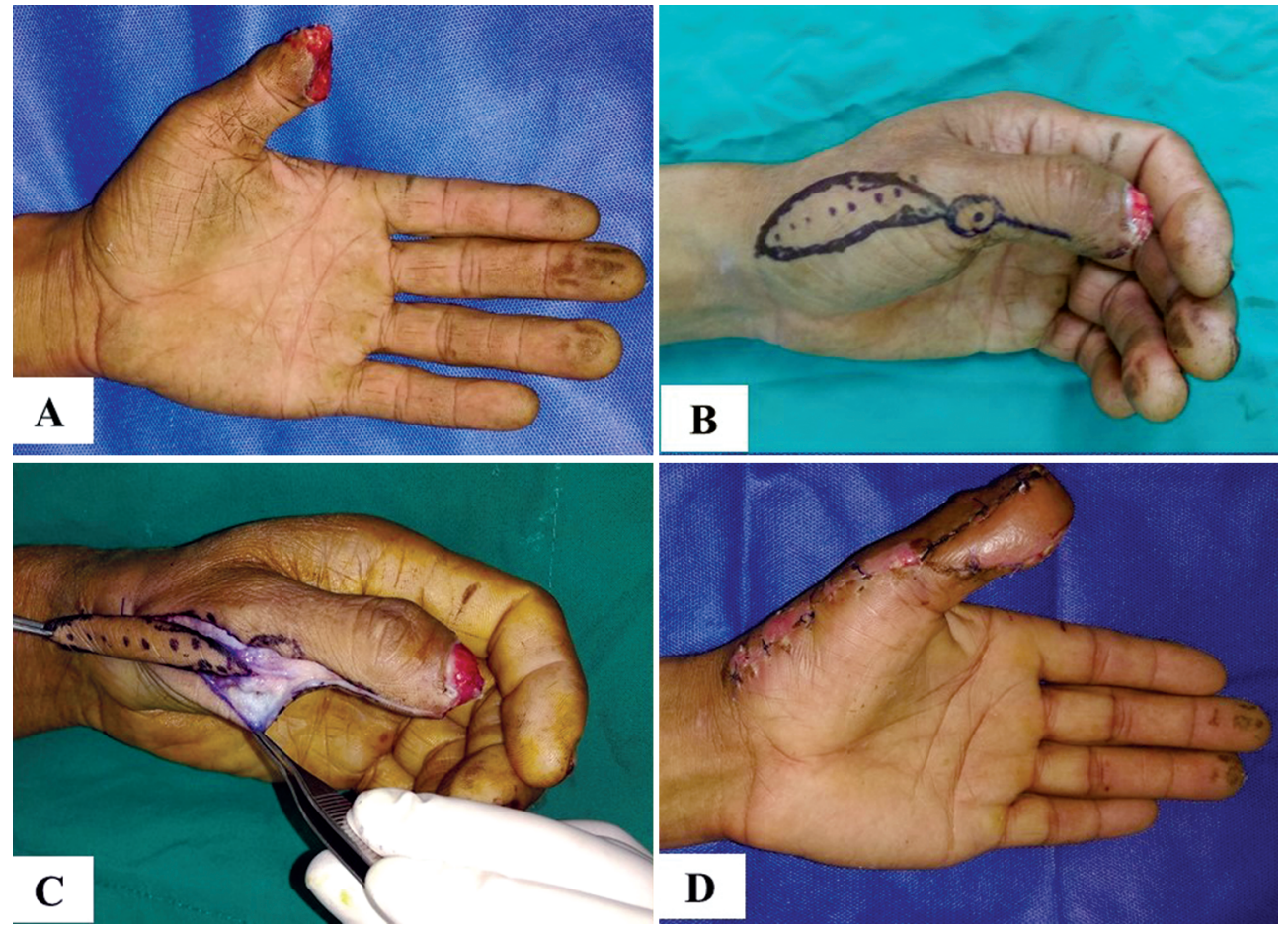

Fig. (4): (A) Preoperative fingertip injury, (B) Preoperative marking of RHDRA flap, (C) Flap raising, (D) Postoperative view 3 weeks later. 


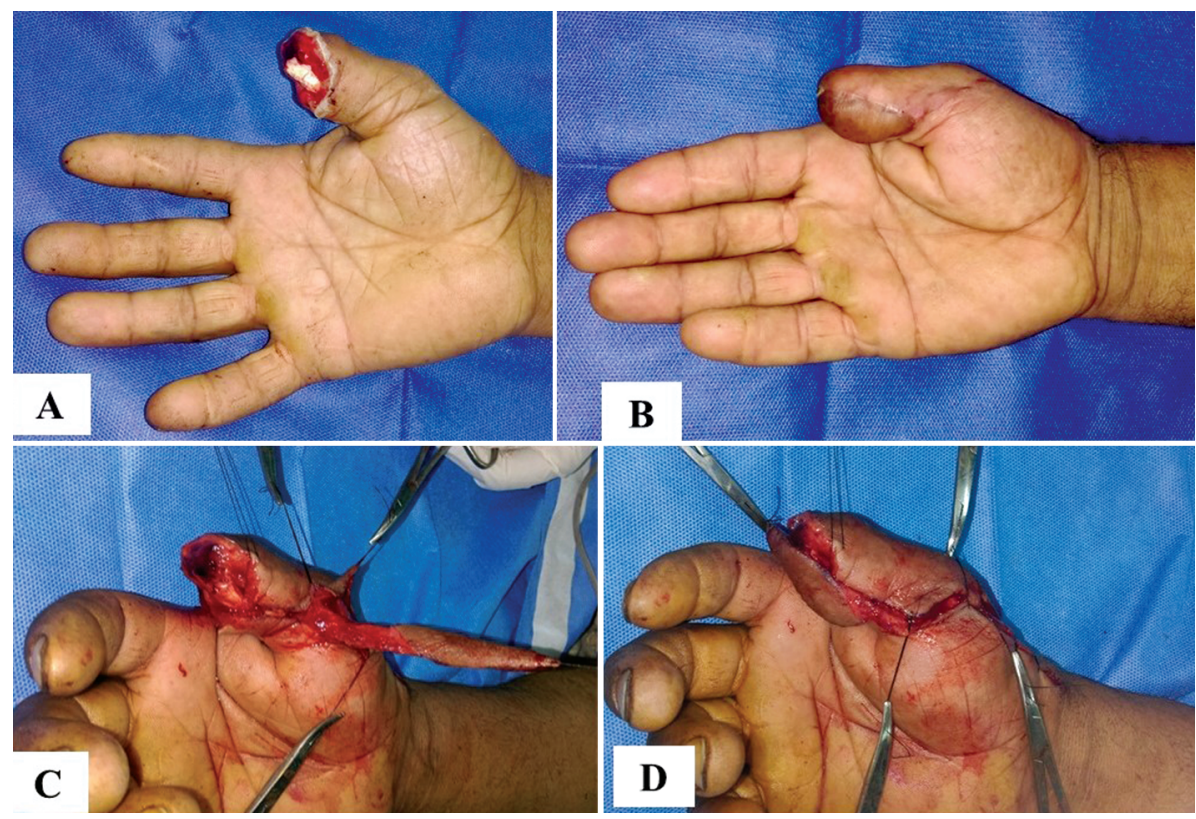

Fig. (5): (A) Fingertip injury with exposed tendon, (B) Postoperative view 3 months later of RHDRA flap, (C) Flap raising, (D) Flap insitting.

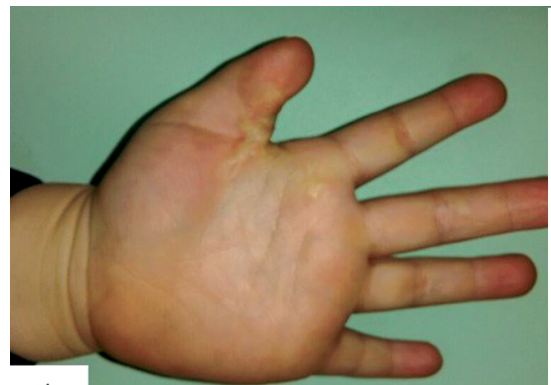

A

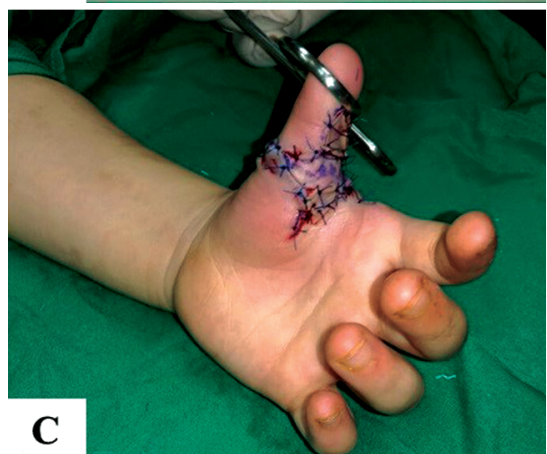

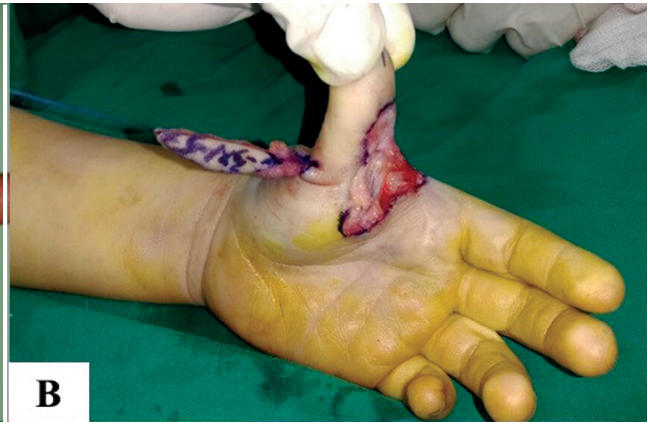

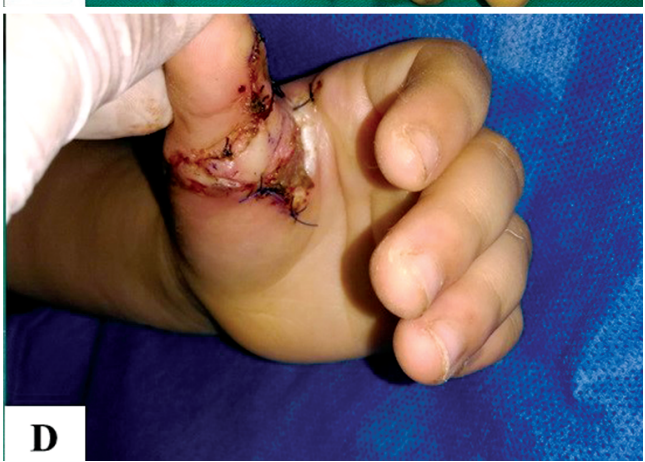

Fig. (6): (A) $1^{\text {st }}$ web space contracture [post burn], (B) Flap raising and contracture release, (C) Intraoperative flap insitting, (D) Postoperative view 2 weeks later.

\section{DISCUSSION}

Reconstruction of hand soft tissue defects, and in particular in the thumb, presents a challenging task in relation to the restricted availability of local tissues. Recent evolves in anatomical research studies and continuous refinements in flap design and harvesting techniques have led to considerable improvement in aesthetic and functional results in thumb soft tissue reconstruction $[4,11]$.
Both of $1^{\text {st }}$ DMCA and RHDRA flaps can provide a versatile, sensate, moderately sized skin paddle on a reliable vascular axis, allowing onestage reconstruction for thumb defects without prolonged immobilization in an inappropriate position and without the need for free flap microsurgical anastomosis [11,12].

The major advantage of the RHDRA flap is its independence from other fingers with inconspicu- 
ous donor-site morbidity, often without skin graft at the donor site. This is critically important in case of mutilating hand trauma which might involve the neighboring index finger, thus reducing the availability of the donor site of heterodigital $1^{\text {st }}$ DMCA [1]. Also the major inconvenience of $1^{\text {st }}$ DMCA flap harvesting; it probably leaves an obvious scar on the dorsum of the hand with mandatory skin graft at the donor site, which may be undesirable, particularly in female patients [12].

As regard the complication; the problem of venous congestion was the mostly occurring one, in only one case in group A and 3 cases in group B. this can be explained as the venous drainage of the concomitant veins of the $1^{\text {st }}$ DMCA is very reliable [8], on the other hand venous outflow in group B takes a random pattern rather than a defined one after the dorsal radial artery in several anatomical dissections [1], we took enough soft tissue around the pedicle to overcome this problem. Also in both group we did not try to identify or skeletonize the vascular pedicle to avoid flap vascularity impairment.

The skin island dimensions [width and length] was statistically larger with the reversed flap than 1 st DMCA flap, beside the versatility of the pivot point, this is allowed coverage of wider and distal thumb defects [8], to overcome this problem, ElKhatib [13] and Gebhart and Meissl [14] had reported in a small number case series; the use of an extended skin island 1st DMCA flap that included the dorsal skin from both the proximal and middle phalanges of the index finger.

In our cases, good recovery of protective sensibility was obtained in both groups, the mean value of static two-point discrimination in group A was 10.66 , which agreed with other searchers such as; with Chang et al., [15] it was 8.12, Muyldermans and Hierner [8] it was 10.57, and with Ege et al., [12] it was 10.8. While in group B, it was 12.6 relatively higher than reported by Moschella, and Cordova [3] which was 9.7, this perhaps because we did the test in the early postoperative period after 6 weeks.

We agreed with the work of Tränkle et al., [16], that the innervated $1^{\text {st }}$ DMCA flap provides immediate sensation, but the main concern was about the dual location phenomenon, and the problem of cortical reorientation. Muyldermans and Hierner [8] mentioned that cortical reorientation takes some time, but is usually complete after 2 years. The incomplete reorientation was reported not to be disturbing and did not interfere with patient's daily activities. If however, on patient demand Foucher [17] had corrected this situation surgically with a technique (débranchement-rébranchement) by dividing the transferred nerve and resuturing it to the original nerve of the thumb.

Moschella, and Cordova [3] also reported that epineural suturing between the cutaneous branch of the radial nerve, enclosed in the flap, and a digital nerve, identified at the recipient site did not improved the sensitivity, they no longer recommended this step.

\section{Conclusion:}

The $1^{\text {st }}$ dorsal metacarpal artery flap had been recognized as a keystone in the reconstruction of thumb defects, on the other hand the reverse homodigital dorsoradial artery flap could be considered another useful alternative reconstructive tool in thumb reconstruction. Both flaps have a constant vascular anatomy with straightforward and easy harvesting technique, and sensate reconstructed pulp area. The RHDRA flap has relatively larger skin island with inconspicuous residual scar at donor site, and less operative time compared to $1^{\text {st }}$ DMCA flap.

\section{REFERENCES}

1- Hrabowski M., Kloeters O. and Germann G.: Reverse Homodigital Dorsoradial Flap for Thumb Soft Tissue Reconstruction: Surgical Technique. J. Hand. Surg., 35A: 659-662, 2010.

2- Chao J.D., Huang J.M. and Wiedrich T.A.: Local hand flaps, journal of the American society for surgery of the hand, 1 (1): 25-44, 2001.

3- Moschella F. and Cordova A.: Reverse Homodigital Dorsal Radial Flap of the Thumb. Hand/Peripheral Nerve. Plast. Reconstr. Surg., 117 (3): 920-926, 2006.

4- Germann G., Sauerbier M., Rudolf K.D. and Hrabowski M.: Management of Thumb Tip Injuries, current concepts. J. Hand. Surg. Am., 40 (3): 614-622, 2015.

5- Azari K. and Lee W.P.A.: Chapter 55: Thumb reconstruction. In: Wolfe S.W., Pederson W.C., Hotchkiss R.N., Kozin S.H. Green's Operative Hand Surgery. $6^{\text {th }}$ ed. Philadelphia, PA: Churchill Livingstone., 1839-1881, 2010.

6- Daniali L. and Azari K.: Reconstruction of a Distal Dorsal Thumb Defect. www.ePlasty.com, Interesting Case, April, 17, 2013.

7- Pistre V., Pelissier P., Martin D. and Baudet J.: Vascular blood supply of the dorsal side of the thumb, first web and index finger: anatomical study. J. Hand. Surg. Br., 26 (2): 98-104, 2001.

8- Muyldermans T. and Hierner R.: First dorsal metacarpal artery flap for thumb reconstruction: A retrospective clinical study. Strat Traum Limb Recon., 4: 27-33, 2009. 
9- De Rezende M.R., Júnior R.M., Cho A.B., Hasegawa O.H. and Ribak S.: Anatomic study of the dorsal arterial system of the hand. Rev. Hosp. Clín. Fac Med. S. Paulo., 59 (2): 71-76, 2004.

10- Earley M.J.: The arterial supply of the thumb, first web and index finger and its surgical application. J. Hand. Surg., 11B: 163-74, 1986.

11- Germann G. and Palm-Bröking K.: First Dorsal Metacarpal Artery Island Flap https://musculoskeletalkey.com/firstdorsal-metacarpal-artery-island, flap/Word Press theme by UFO themes. 2016.

12- Ege A., Tuncay I., and Ercetin O.: Foucher's first dorsal metacarpal artery flap for thumb reconstruction: Evaluation of 21 cases, IMAJ., 4: 421-423, 2002.

13- El-Khatib H.A.: Clinical experiences with the extended first dorsal metacarpal artery island flap for thumb re- construction. J. Hand .Surg. [Am.], 23 (4): 647-652, 1998.

14- Gebhart B. and Meissl G.: An extended first dorsal metacarpal artery neurovascular island flap. J. Hand. Surg., 20B: 529-531, 1995.

15- Chang S.C., Chen S.L., Chen T.M., Chuang C.J., Cheng T.Y. and Wang H.J.: Sensate first dorsal metacarpal artery flap for resurfacing extensive pulp defects of the Thumb. Annals of Plastic., 53 (5): 449-455, 2004.

16- Tränkle M., Sauerbier M., Heitmann C. and Germann G.: Restoration of thumb sensibility with the innervated first dorsal metacarpal artery island flap. J. Hand .Surg. Am., 28 (5): 758-766, 2003

17- Foucher G. and Braun J.B.: A new island flap transfer from the dorsum of the index to the thumb. Plast. Reconstr. Surg., 63: 344-349, 1979. 